\title{
Computer Visualization of Julia Sets for Maps beyond Complex Analyticity
}

\author{
Alexey Toporensky ${ }^{1, *}$ and Ivan Stepanyan ${ }^{2}$ \\ ${ }^{1}$ Sternberg Astronomical Institute, RU-119234, Moscow, Russian Federation \\ ${ }^{2}$ Mechanical Engineering Research Institute of the Russian Academy of Sciences, RU-101990, Moscow, Russian Federation
}

\begin{abstract}
Using the c omputer pr ogram c reating $J$ ulia $s$ ets for $t$ wo-dimensional $m$ aps $w e ~ h$ ave made computer animation showing how Julia sets for the Peckham map alters when the parameter of the map is changing. The Peckham map is a one-parameter map which includes the complex map $\mathrm{z}=\mathrm{z}^{\wedge} 2+\mathrm{c}$, and is nonanalytical for o ther values of the p arameter. Computer a nimation of Julia fractal s ets allows s eeing how patterns typical for complex maps gradually destroy.
\end{abstract}

\section{Introduction}

Iterations o f co mplex a nalytic maps ap pears to be a wonderful s ource of fractal s tructures, i nteresting from both mathematical and aesthetic viewpoints. Already the simplest map

$$
\mathrm{z}=\mathrm{z}^{2}+\mathrm{c}
$$

where $\mathrm{c}$ i s a co nstant co mplex $\mathrm{n}$ umber co ntains $\mathrm{v}$ ery complicated d ynamics, and Julia sets for certain values of the $\mathrm{p}$ arameter $\mathrm{c}$ r epresent a $\mathrm{t}$ rue co mputer ar $\mathrm{t}$ ( $\mathrm{s}$ ee examples in [1]).

There are several methods to generalize the map (1). One way is to retain the complex analyticity and consider $\mathrm{p}$ olynomial or $\mathrm{t}$ ranscendent maps. T here is a well $\mathrm{d}$ eveloped $\mathrm{m}$ athematical $\mathrm{t}$ heory for $\mathrm{s}$ uch kind of maps ( see, for ex ample, Milnor's 1 ectures [2] an d references therein). Julia sets for such maps has roughly the same structure, though can get additional symmetries in the p olynomial cas e. $\mathrm{P}$ articular cas e of $\mathrm{Z}$ hukovski map have been considered in [3], for more general forms of maps within complex analyticity see, for example, [4]. Among $r$ ecent $r$ esults we $c$ an i ndicate $t$ he $p$ aper [5] where it was shown that the Julia set of a transcendental meromorphic map with at most finitely many pol es cannot be contained in any finite set of straight lines and the pa per [6] which de scribes t he $c$ onditions of existence of Sierpiński carpet Julia sets and estimation of it's Hausdorff dimension.

We should also note that in some cases Julia set can bee smooth, see [7] which gives the examples of smooth Julia s ets, a mong th em: a c ircle, a s egment, a $n$ in finite interval, a $\mathrm{s}$ traight li ne, a nd th e c omplex p lane. I $t$ is shown that the functions s tudied in [7] ar e ch aotic o n their J ulia sets. An i nteresting $g$ eneralization o $\mathrm{f}$ Mandelbrot and Julia sets to hyperbolic dynamics can be found in the paper [8].
Abandoning c omplex a nalyticity gi ves $r$ ise $t$ o ne w events, an $\mathrm{d} t$ he co rresponding mathematical theory is developing i ntensively a $t \mathrm{t}$ he $\mathrm{p}$ resent $\mathrm{t}$ ime [9]. Visual patterns of analogs of the Mandelbrot set usually differ qualitatively from the fractal s tructure of the "true" Mandelbrot s et, which can $b$ e s een cl early for $t$ he "burning ship" map [10]. This map differs from (1) only by the presence of moduli, a nd it is e nough to convert the $\mathrm{M}$ andelbrot $\mathrm{s}$ et i nto a morphous s tructure with $\mathrm{thin}$ linear ba nds. I $\mathrm{n}$ a br oader $\mathrm{c}$ ontexts, bot $\mathrm{h} \mathrm{c}$ omplex analytic $\mathrm{m}$ aps and the "burning $\mathrm{s}$ hip" $\mathrm{m}$ ap represent particular cases of two-dimensional real maps, which can be derived from the complex form by extracting real and imaginary parts. In principle, one-dimensional real maps also can have a co mplicated ch aotic $n$ ature ( see, for example, [11]), though they are less interesting from a viewpoint of a computer art. form

In this form the complex analytic map (1) has the

$$
\begin{aligned}
& x=x^{2}-y^{2}+R(c), \\
& y=2 x y+I(c)
\end{aligned}
$$

and in the "burning ship" map the equation (2) remains the same, while (3) becomes

$$
\mathrm{y}=2(x)(y)+I(c)
$$

\section{Results}

For Mandelbrot and Julia s ets for two-dimensional real maps these long quasi-parallel $b$ ands an $d$ o ther 1 inear structures ap pear to b e r ather typical ( see e xamples in [1]). This means that complex analytic maps have some special $\mathrm{p}$ roperties. $\mathrm{F}$ ormally, th ey $\mathrm{d}$ emand $\mathrm{C}$ auchyRiemann $\mathrm{c}$ ondition to $\mathrm{b}$ e satisfied, which le ads to absence of s addle p oints and ab sence of ap pearance or disappearance of $\mathrm{f}$ ixed poi nts with $\mathrm{c}$ hange of $\mathrm{t}$ he

e-mail: atopor@rambler.ru 
parameter of the map (1) [9]. The letter follows directly from $t$ he fundamental theorem of algebra, the former follows from the fact that a complex multiplication is a combination of stretching and rotation, while a map in a saddle point stretches in one dimension and contracts in another one.

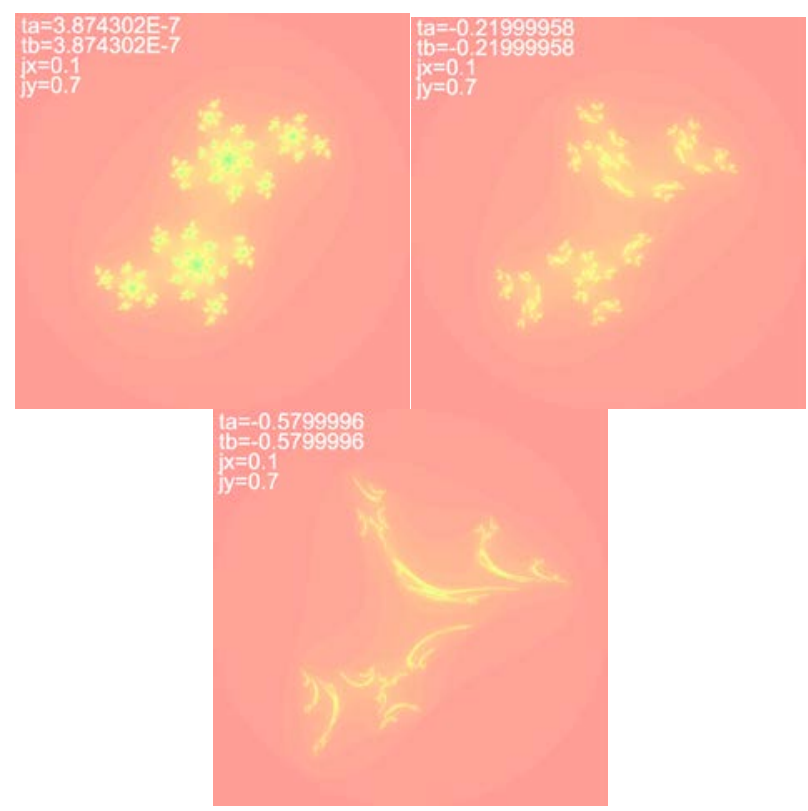

Fig.1. Three frame from the animation showing gradual change of Julia set with the deviation of complex analyticity.

Parameters of the map is shown in the pictures. Here jx is real, and jy is the imagery parts of $c$, ta and tb represents a and b (they are equal for the Packham map). Left panel is the analytic map

In $t$ he $p$ aper [ 9] $\mathrm{B}$. Peckham $d$ escribed $h$ ow $t$ his properties $\mathrm{h}$ ave $\mathrm{b}$ een 1 ost when $\mathrm{a}$ map $\mathrm{b}$ ecomes nonanalytical. For this purpose the map

$$
\mathrm{z}=\mathrm{z}^{2}+\mathrm{c}+\mathrm{a}
$$

have been used. It coincides with ( 1 ) for $\mathrm{a}=0$, and is not analytic for any nonzero a. The goal of our paper is to describe typical $v$ isual $f$ orms of $t$ he Peckham $m$ ap (gradual d eveloping o $\mathrm{fl}$ inear structures through the classical Mandelbrot set for the Peckham map have been considered in [12].

Strictly speaking, $\mathrm{t}$ he $\mathrm{r}$ esults $\mathrm{p}$ resented $\mathrm{b}$ elow ar e related to filled Julia sets, i.e. sets of i nitial points such that iterations of $\mathrm{t}$ he map (5) $\mathrm{r}$ emake bou nded from above. They are shown in white. Initial points from other regions leave the vicinity of the coordinate origins (we set it to a radius $10 \mathrm{~d}$ isc in o ur numerical s tudies), the color encodes the escape time needed.

Keeping i $\mathrm{n}$ mind $\mathrm{p}$ ossible $\mathrm{g}$ eneralizations of th $\mathrm{e}$ Packham map, we realize more general map

$$
\begin{aligned}
& \mathrm{x}=\mathrm{x}^{2}-y^{2}+R(c)+\mathrm{ax}, \\
& \mathrm{y}=2 \mathrm{xy}+I(c)-\mathrm{by} .
\end{aligned}
$$

However, $\mathrm{f}$ or $\mathrm{t}$ he a nimations we have $\mathrm{c}$ hosen $\mathrm{t}$ he original Packham $\mathrm{m}$ ap, $\mathrm{w}$ ith $\mathrm{a}=\mathrm{b}$. A nimations $\mathrm{s}$ how gradual change of Julia sets with changing parameter a.
Visual patterns of Julia sets rapidly loose features known from $t$ he co mplex a nalytic $d$ ynamics, $b$ ecoming $r$ ather prolonged ( and, u sually, 1 ess aes thetically at tractive). Figure $1 \mathrm{r}$ epresent $\mathrm{t}$ hree $\mathrm{f}$ rames from o ur a nimation, showing the $t$ ransition from $c$ omplex a nalytic $p$ attern (left figure) to quasi-linear structures (right figure).

Some examples of Julia sets for the map with a not equal to be are presented in Fig.2. To our mind, current results i ndicate t hat "aesthetic maximum" i s $\mathrm{r}$ ealized usually for the co mplex an alytic map with $a=b=0$ i $n$ comparison with non-analytic maps.

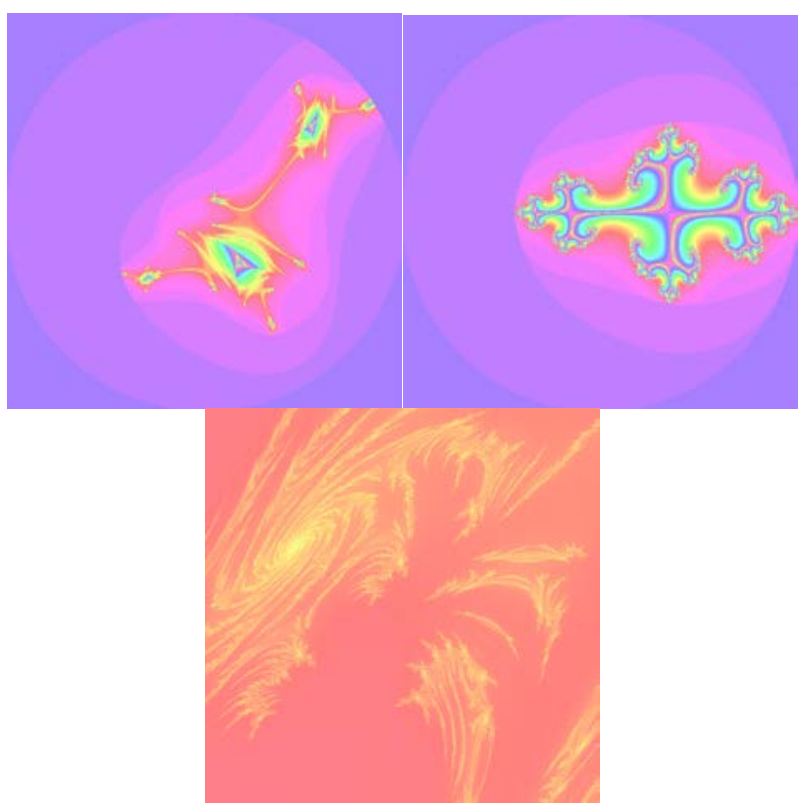

Fig.2. Examples of diagrams for general case of different parameters $a$ and $b$. From left to right: $a=-0,75, b=1,6, x=0,74$, $y=0,97 ; a=1,14, b=1,39, x=0,28, y=0,01 ; a=-0,6, b=0,3, x=-$ $0,7, y=0,3$

\section{Discussion and Conclusions}

We should however note that complex analytic dynamics is no $t$ unique in p roducing visually attractive $\mathrm{J}$ ulia s ets among two-dimensional real maps. Another example is given by the "burning ship" map. It's Mandelbrot set is rather typical for real maps, however, Julia sets represent unexpectedly interesting constructions, though probably less attractive than for analytic maps.

Fig. 3 represents three frame from our visualization of Julia s ets of the "burning s hip" map. Adding the term proportional to $\bar{Z}$ into the right hand side of the equations for "burning s hip" map s hould d estroy s uch s tructures. This is illustrated in Fig.4 created for the map

$$
\begin{aligned}
x=x^{2}-y^{2}+R(c)+a x, \\
y=2(x)(y)+I(c)-b y .
\end{aligned}
$$

The Fig. 4 shows four frames from the corresponding animation. 


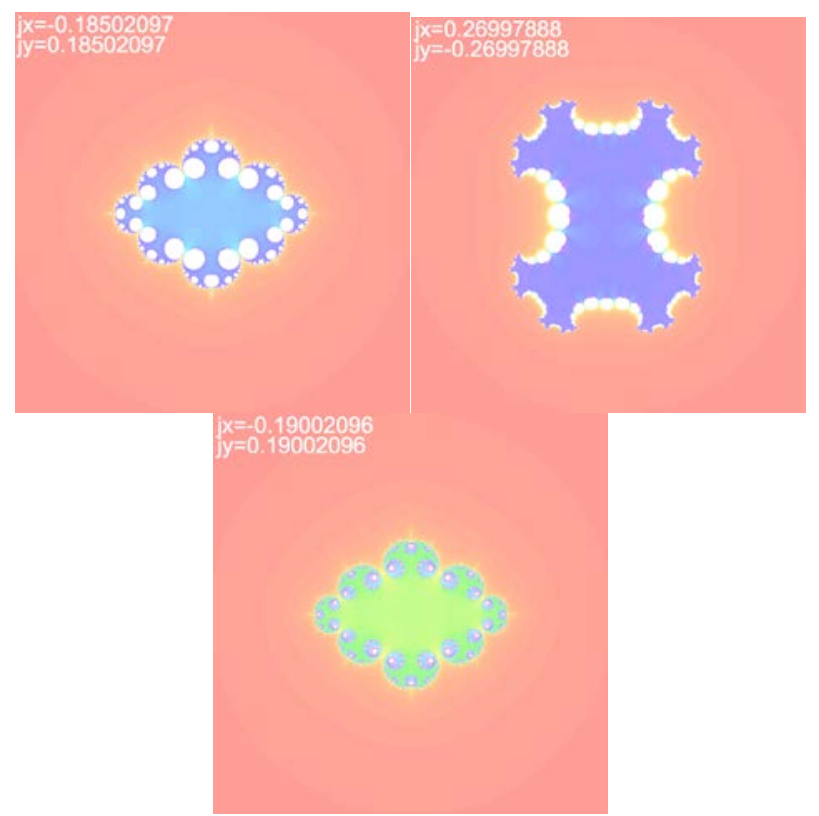

Fig.3. Examples of Julia sets for the "burning ship" map

We have co nsidered the influence of co mplex nonanalyticity up on visual forms o $\mathrm{fJ}$ ulia $\mathrm{s}$ ets o $\mathrm{f}$ corresponding maps. For the Packham map the Julia sets rapidly evolve from known "classical" forms of the map (1) to forms typical for two-dimensional real maps with increasing of modulus of the parameter a. Already for $|\mathrm{a}|$ about $0.2 \mathrm{t}$ races of co mplex an alyticity have $\mathrm{b}$ ecome hardly d etectable. $W$ e can e ven c laim t hat a $g$ eneral aesthetic attraction falls rapidly with deviating from complex analyticity ( recognizing that such statement is inevitably subjective and nonformalizable) confirming a special $r$ ole o f co mplex a nalytical maps i n co mputer graphics.

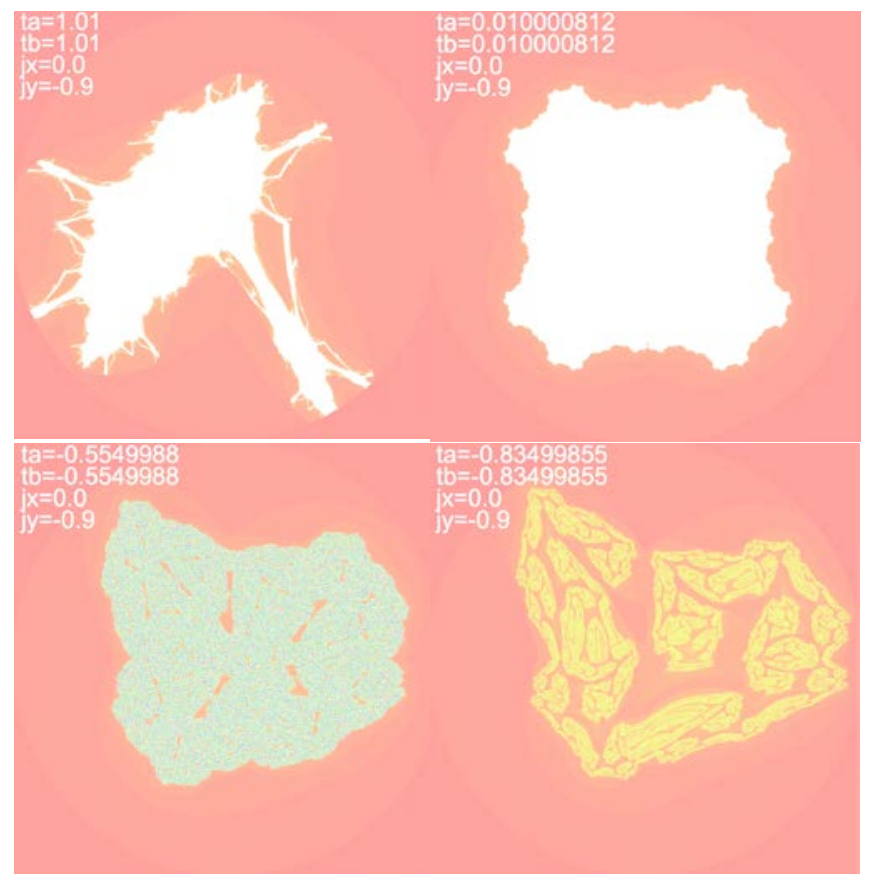

Fig.4. Diagrams for "deformed burning ship" map (8)-(9)
Nevertheless, examples of Julia sets for non-analytic map $(2,4)$ show that the Cauchy - Riemann conditions are not strictly necessary for Julia sets to be aesthetically attractive (which is also interesting since the Mandelbrot set for the map $(2,4)$ has totally different visual forms). This means $t$ hat e ven for $r$ ather $\mathrm{s}$ imple cas e o $\mathrm{ft}$ wodimensional maps the connection between mathematical struture and aesthetic a ttraction is not so tight, and the search for aesthetically attractive Julia sets should not be bound only to complex analytic maps, despite their special role in computer art.

\section{References}

1. H.-O. Peitgen, P.H. Richter, The Beauty of Fractals (Springer-Verlag, 1986)

2. J. M ilnor, Dynamics $i$ $n$ One $C$ omplex $V$ ariable (Vieweg, 1999)

3. J.B. M ugiraneza, I .J. I mage, $\mathrm{G}$ raphics an $\mathrm{d} \mathrm{S}$ ignal Processing, 5, 61-70 (2012)

4. Sh. Agarwal, I .J. C omputer N etwork a nd Information Security, 4, 1-9 (2017)

5. C. $\mathrm{C}$ ao, $\mathrm{Y}$. W ang, Acta $\mathrm{M}$ ath $\mathrm{S}$ ci, 40, $903-909$ (2020) https://doi.org/10.1007/s10473-020-0401-5

6. Y. F u, F . Y ang, Math. Z ., 294, 144 1-1456 (2020) https://doi.org/10.1007/s00209-019-02319-4

7. V.S. Sekovanov, J Math Sci, 245, 202-216 (2020)

8. V. Blankers, et al., Fractal and Fractional, 3(1) (2019) https://doi.org/10.3390/fractalfract3010006

9. B. P eckham, Int. J. Bifurcation a nd $\mathrm{C}$ haos, $\mathbf{8}(73)$ (1997)

10. M. M ichelitsch, $\mathrm{O}$. R ossler, $\mathrm{C}$ omputers an d Graphics, 16(435) (1992)

11. Sh. X u, Y . W ang, Y . G uo, C. W ang, I .J. I mage, Graphics and Signal Processing, 1, 61-68 (2010)

12. A. T oporensky, Quasi-Mandelbrots ets $f$ or perturbed c omplex analytic maps:visual pattern $\mathrm{s}$ (2008) arXiv:0807.1667 